\title{
Search for Dark Matter Using Upward-going Muons in NOvA
}

\author{
C. Principato*, C. Group, S. Kurbanov, R. Mina, Y. Oksuzian \\ University of Virginia \\ E-mail: cp2vk@virginia.edu
}

\section{A. Tsaris, L. Aliaga, P. Ding, A. Norman \\ Fermi National Accelerator Laboratory}

\begin{abstract}
The NOvA collaboration has constructed a 14,000 ton, fine-grained, low-Z, total absorption tracking calorimeter at an off-axis angle to an upgraded NuMI neutrino beam. This detector, with its excellent granularity and energy resolution and relatively low-energy neutrino thresholds, was designed to observe electron neutrino appearance in a muon neutrino beam, but it also has unique capabilities suitable for more exotic efforts. In fact, with an efficient upward-going muon trigger and sufficient background suppression offline, NOvA will be capable of a competitive indirect dark matter search for low-mass WIMPs. Interesting atmospheric neutrino measurements should also be possible. The cosmic ray muon rate at the NOvA far detector is about $150 \mathrm{kHz}$ and provides the primary challenge for triggering and optimizing such a search analysis.
\end{abstract}

38th International Conference on High Energy Physics

3-10 August 2016

Chicago, USA

${ }^{*}$ Speaker. 


\section{Timing resolution of the detector}

At NOvA for non-beam exotic physics searches, so-called data-driven triggers [1] are required to select events of interest.

For the upward-going muon trigger, the idea is to first select high-quality muon tracks. Once we have a sample of well-reconstructed muon tracks we use the timing information of all of the hits on each track to estimate directionality and this can be used to reduce the rate by at least the required four orders of magnitude [2].

Each time measurement has an uncertainty, which varies with the amount of energy deposited. The time uncertainty on a given hit from a reconstructed muon track affects the determination of track directionality, so a parameterization of uncertainty in terms of energy deposition is necessary for the timing-based trigger. Upgrading the Far Detector readout from "singlepoint" to "multipoint" readout improved the single-hit timing resolution from about $125 \mathrm{~ns}$ to better than $15 \mathrm{~ns}$.

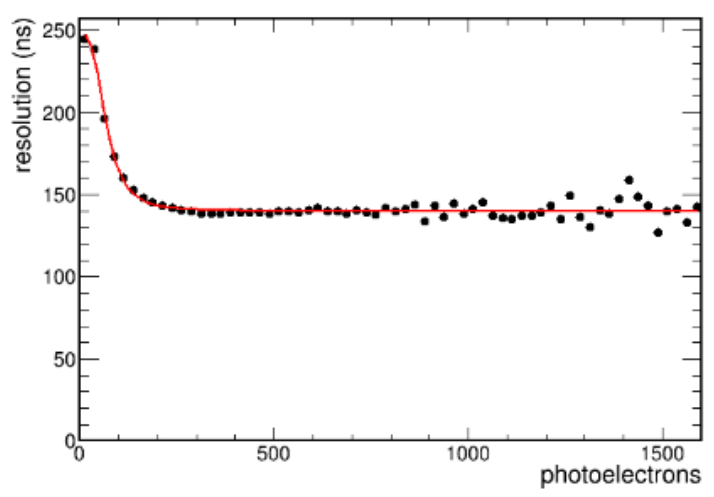

(a) single-point

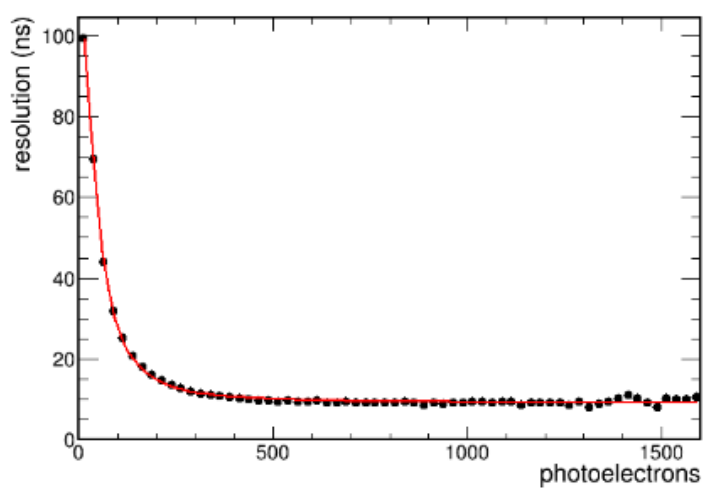

(b) multi-point

Figure 1: Single-hit timing resolution as observed in NOvA far detector data with four-point readout, before (left) and after (right) fine timing implementation. See Ref. [5] for more details.

A further improvement in the timing resolution has been achieved by using a new pulse shape function with a more realistic preamplifier response (See Ref. [3] and Ref. [4] for more information).

$$
f(t)=H F I\left(\frac{e^{\frac{t-t_{0}}{F}}}{(I-F)(F-R)}-\frac{e^{\frac{t-t_{0}}{I}}}{(I-F)(I-R)}+\frac{e^{\frac{t-t_{0}}{R}}}{(I-R)(R-F)}\right)+C
$$

where $H, F, I, R, C$ are respectively the Pulse height, Fall time, Pre-Amplification time, Rise time and Baseline.

Recently, we updated the rise and fall times used to parameterize the function used in these fits [9]. In the LLR calculation we use a paramatrization of the single-hit timing resolution as a function of ADC value.

\section{Discriminating upward-going from downward-going muons}

The time expected and observed for a single hit is to produce the distribution of the expected v/s observed time for each track as shown in Fig. ??. The distribution is produced using a reconstructed upward-going muon track simulated with WIMPSIM [6, 7]. The slope values for cosmics 
and WIMPSIM MC samples are consistent with the downward- and upward-going hypothesis, respectively. Therefore, we can fit the time distribution on Fig. ?? with fixed values of slopes. For the upward-going track the fit with the slope constrained to " 1 " results in a good $\chi^{2}$ probability value of the fit, $P_{\uparrow}$. However the fit with slope of “-1" yields a low probability value, $P_{\downarrow}$. Using the probability values from the fits with the fixed slope value, we can form a log-likelihood ratio (LLR):

$$
L L R=\log \left(\frac{P_{\uparrow}}{P_{\downarrow}}\right)
$$

A cut on LLR slightly above zero will reduce the cosmic background by the desired amount while preserving a high signal acceptance.

The LLR yields better performance for cosmic background rejection for the same signal acceptance in the regime where the cosmic rejection is sufficient (at least four orders of magnitude), compared to a cut on the best-fit slope.

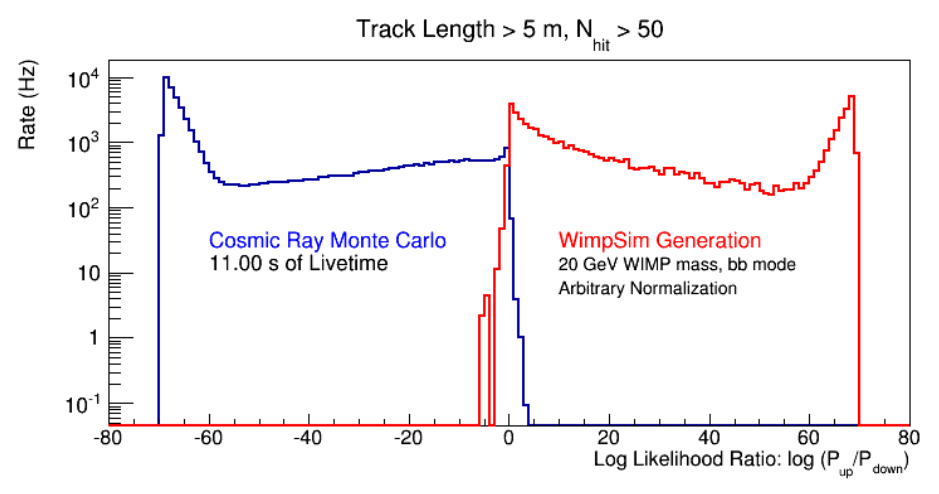

Figure 2: The LLR distributions for cosmics (red) and WIMPSIM (blue) MC samples. Note that only tracks longer then $5 \mathrm{~m}$ and with more than 50 hits are included.

\section{LLR as final discriminant}

In addition to the basic quality cuts, several cuts are designed to select upward-going events (see Table 1.

\begin{tabular}{lll}
\hline Cut & Cut value & Description \\
\hline chi 2 & 1.5 & Fit $\chi^{2} / \mathrm{NDF}$ of time distribution \\
ProbUp & 0.0001 & $\chi^{2} / \mathrm{NDF}$ for the slope=1 fit \\
LLR & 5.0 & LLR of time distribution for all hits \\
LLRX & 3035985 & LLR in the X-view (3D-matched) \\
LLRY & 3035985 & LLR in the Y-view (3D-matched) \\
\hline
\end{tabular}

Table 1: Directionality cuts

The efficiency for these cuts for upward-going muons can be estimated by inverting them and looking at their effect on downward-going cosmic-ray muons. In order to do that, we first apply clean up cuts on the sample and then we apply each cut separately. 
Figure 3 shows how the LLR distribution changes as base cuts are applied. Actually, this is the inverted LLR:

$$
L L R_{\text {invert }}=\log \left(\frac{P_{\downarrow}}{P_{\uparrow}}\right)
$$

Because it is the inverted LLR, downward going tracks are represented by positive values on the Fig. 3. You can see that as the cleanup cuts are applied, especially track length, the LLR distribution becomes much more shifted to high values. For long tracks, $>10 \mathrm{~m}$, the LLR can be used to select the correct direction of tracks with high confidence.
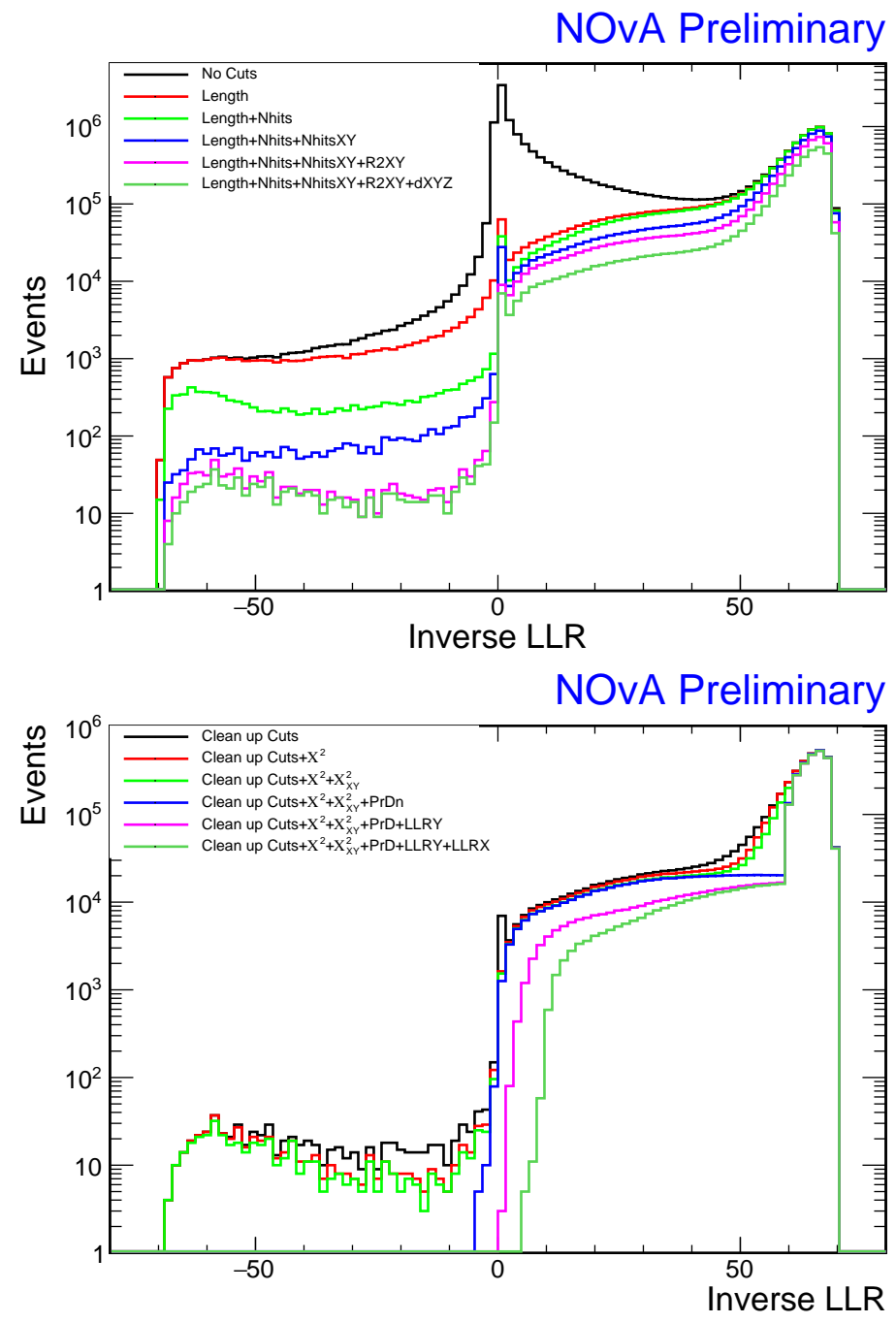

Figure 3: The figure shows how the LLR distribution varies as cleanup cuts are applied (top) and as timing cuts are applied (bottom). This is the inverted LLR distribution for the cosmic sample.

\section{Preliminary Results}

The signal efficiency has been calculated from cosmic studies and is $\approx 30 \%$.

What we found by looking at the upward-going muon sample, were 33 events in the background 
region, and 24 in the twilight region, where 23 were expected. MonteCarlo studiess needs to be done before to look at the signal region.

\section{References}

[1] M. Fischler, C. Green, J. Kowalkowski, A. Norman, M. Paterno and R. Rechenmacher, J. Phys. Conf. Ser. 396, 012020 (2012).

[2] R. Mina et al., âĂIJImplementation of an upward-going muon trigger for indirect dark matter searches at the NOÎj $;$ A far detector.âĂI CHEP2015, Okinawa, Japan, 13-17 April, 2015. Pre-print. Update me!!

[3] NOvA Document Database: Note 13577

[4] NOvA Document Database: Note 13779

[5] E. Niner, Ph.D. Thesis, Indiana University, 2015

[6] M. Blennow, J. Edsjo and T. Ohlsson, JCAP 0801, 021 (2008).

[7] J. Edsjo, WimpSim Neutrino Monte Carlo, http://www.fysik.su.se/ edsjo/wimpsim/.

[8] Y. Oksuzian, C. Group, R. Mina, M. Frank, A. Norman, and E. Fries NOvA Document Database: Note 12116

[9] C. Principato, NOvA Document Database: Note 15047

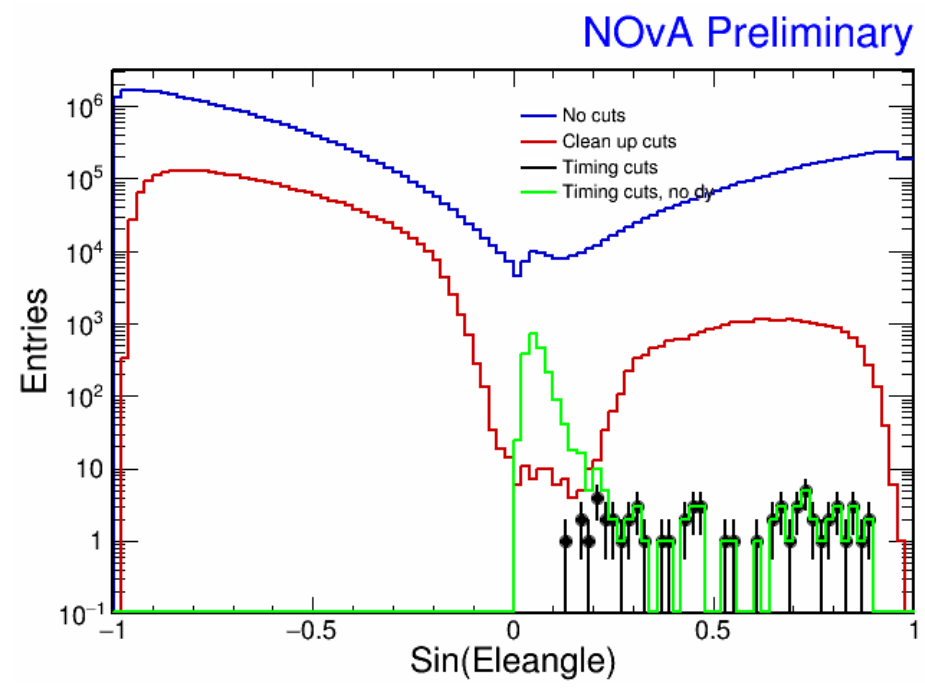

Figure 4: The distribution of sine of the elevation angle for each track in the timing-based candidate subsample (red) and all tracks including those excluded from the subsample (blue). Almost all candidates have an elevation angle near 0, indicating they are nearly parallel with the ground. A negative elevation angle indicates a downward-going track, while positive indicates an upward-going track. By cutting on the length of the track in the y-direction the near-horizontal cosmic-muon-induced background is eliminated. 